\title{
Compositional Analysis of Adsorbed Organic Aerosol on a Microresonator Mass Sensor
}

\author{
Arthur T. Zielinski ${ }^{1}$. Steven J. Campbell ${ }^{1}$ Ashwin A. Seshia ${ }^{2} \cdot$ Roderic L. Jones $^{1} \cdot$ Markus Kalberer $^{1} \cdot$ Chiara Giorio $^{1,3}$
}

Received: 3 April 2018 / Revised: 17 June 2018 / Accepted: 18 June 2018 / Published online: 25 June 2018

(c) The Author(s) 2018

\begin{abstract}
Aerosol mass measurements are a key air pollution parameter that is regulated in most countries. Beyond mass measurements, the precise composition of the aerosol is essential in identifying sources and impacts on health and climate. The conventional method for simultaneously quantifying mass and composition is to collect aerosol onto filter or impactor samples followed by laboratory analysis. This approach requires long collection times—-providing poor time resolution for mass measurementsand long sample preparation prior to analysis. The first limitation can be circumvented with microresonators, which are novel particulate mass sensors with high mass sensitivities and time resolutions. In addition, direct surface analysis techniques, like liquid extraction surface analysis mass spectrometry (LESA-MS), shorten sample preparation times. This work combines, for the first time, the high time resolution mass measurements of a microresonator with the integrated compositional analysis of LESA-MS. Laboratory-produced secondary organic aerosol were collected onto a microresonator via impaction with LESA-MS being used to analyze the chemical composition afterwards. The results were compared with classic filter extraction methods and literature with the final spectra matching the expected reaction products. The combined technique demonstrates an extension to current microresonator applications and illustrates their potential for ambient aerosol studies.
\end{abstract}

Keywords LESA-MS $\cdot$ Aerosol characterization $\cdot \alpha$-pinene SOA $\cdot$ MEMS $\cdot$ Resonator $\cdot$ Bulk acoustic wave

\section{Introduction}

Recently there has been a push towards small sensors for online measurement of aerosol concentrations, dominated by a range of optical methods (Steinle et al. 2015; Grimm and Eatough 2009; Holstius et al. 2014). The push has been driven by the desire for large, simultaneous pollution studies (such as sensor networks (Broday and Citi-Sense Project Collaborators 2017) or personal monitoring (Steinle et al.

Arthur T. Zielinski

arthur.zielinski@atm.ch.cam.ac.uk

$\triangle$ Chiara Giorio

chiara.giorio@unipd.it

1 Department of Chemistry, University of Cambridge, Lensfield Road, Cambridge CB2 1EW, UK

2 Department of Engineering, Nanoscience Centre, University of Cambridge, 11 JJ Thomson Avenue, Cambridge CB3 OFF, UK

3 Present Address: Dipartimento di Scienze Chimiche, Università degli Studi di Padova, via Marzolo 1, 35131 Padua, Italy
2013)) that require sensors to be small and low cost. Small sensors focus on measuring particle mass, a commonly regulated metric (European Parliament and Council of the European Union 2008), which has often been correlated to major health concerns (Harrison and Yin 2000; Harrison et al. 2010; Raaschou-Nielsen et al. 2013; Xing et al. 2016). Traditional methods, namely filters and impactors, persist in many scientific studies due to their capability of providing additional analysis opportunities such as chemical characterization (Kourtchev et al. 2013; Amato et al. 2016). These traditional methods are limited by their overall bulkiness and low mass sensitivity which requires long collection times (on the order of hours to days) and causes lower time resolutions. However, a new online method of aerosol measurement using microresonators could negate the need for having filter samples in tandem with other online measurement systems.

Microresonators, which are micro-scale resonant masses, have seen growing popularity in a range of applications serving as reference oscillators (Abdolvand et al. 2008), signal filters (Piazza et al. 2007), and sensors (e.g., temperature (Jha et al. 2007), acceleration (Zou et al. 
2014), and mass). Their development as particulate mass sensors is principally due to their high sensitivity, direct mass measurements (Wasisto et al. 2016; Maldonado-Garcia et al. 2016; Paprotny et al. 2013; Zielinski et al. 2016). They offer an alternative to optical particle counting methods that only infer mass and fail to measure ultrafine $(<100 \mathrm{~nm}$ diameter) particles (Heim et al. 2008; Wang et al. 2015), potentially the most influential for human health (Harrison and Yin 2000). Similar to tapered element oscillating microbalances (TEOMs) (Allen et al. 1997) - a popular instrument for air quality monitoring (Steinle et al. 2015) - microresonators operate based on mass adsorption which slows their frequency of vibration and can be related to mass with a linear scaling factor (Lee et al. 2007). A separate method of particle collection and sizing is required, however, since microresonators lack any inherent sizing abilities. Implemented methods include inertial impaction (Maldonado-Garcia et al. 2016; Zielinski et al. 2016), thermophoresis (Paprotny et al. 2013), and electrophoresis (Wasisto et al. 2016). The requirement of particle deposition limits the lifetime of the device (Mehdizadeh et al. 2017), but in turn enables the potential for additional analysis on the collected aerosol similar to filter or conventional impactor samples. After the additional analysis is complete, microresonators can be regenerated using a number of cleaning methods presented in the literature (Mehdizadeh et al. 2017; Wasisto et al. 2013, 2013, 2015; Zielinski et al. 2017) and reused for further measurements.

Filter samples are commonly analyzed for the chemical composition of collected aerosol (Kourtchev et al. 2013; Chow and Watson 2007). For detailed organic composition analysis, the typical method for sample preparation consists of solvent extraction, generally with the aid of sonication, followed by evaporating the solvent and concentrating the samples prior to analysis (Kourtchev et al. 2013; Romonosky et al. 2014; Ohno et al. 2016; Wang et al. 2016; Nguyen et al. 2011). This approach has been validated for targeted analysis but risks modifying the composition of the sample and introducing artefacts in non-targeted analysis (Kourtchev et al.
2013; Mazzoleni et al. 2012; Bateman et al. 2008; Miljevic et al. 2014). Direct analysis methods, such as desorption electrospray ionization (DESI) and liquid extraction surface analysis (LESA) mass spectrometry (MS), circumvent these issues as they do not require the same degree of sample preparation. Direct methods are also faster, on the order of seconds to minutes, which lowers the risk of altering the samples through additional chemical reactions exhibited by conventional extraction methods. DESI-MS and LESA-MS have already been applied to the analysis of both ambient and laboratory-generated aerosol samples (Chen et al. 2008; Li et al. 2009, 2009; Laskin et al. 2010; Fuller et al. 2012; O'brien et al. 2013). However, these techniques have not seen widespread use in the field of atmospheric aerosol because they pose challenges in the analysis of filter samples as the extractive solvent is often absorbed into the filter. LESA-MS, therefore, requires that traditional filters are replaced by different materials. For example, Fuller et al. (2012) collected time-resolved ambient aerosol onto Mylar strips using a rotating drum impactor.

A traditional filter can similarly be replaced with a microresonator to offer a platform for including integrated compositional information (via LESA-MS) with online particulate mass measurements (via measured frequency shifts). We investigated the capabilities of this approach for the first time using secondary organic aerosol produced in laboratory experiments from ozonolysis of $\alpha$-pinene. The compositional results were compared to an equivalent filter sample and traditional samples work-up.

\section{Materials and Methods}

\subsection{Aerosol Production and Collection}

Secondary organic aerosol (SOA) was produced by mixing a $0.2 \mathrm{~L} / \mathrm{min}$ synthetic air (Zero grade, BOC) flow over 1 or $0.5 \mathrm{~mL}$ of $\alpha$-pinene (98\%, Sigma-Aldrich) with 1.0 or $0.2 \mathrm{~L} /$ min flow of ozone for collection with a microresonator or

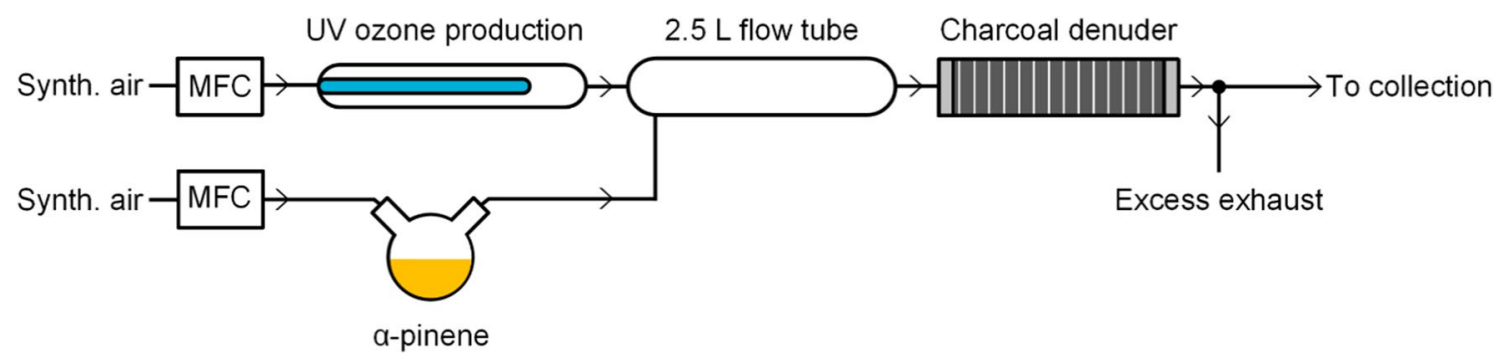

Fig. 1 Method of producing secondary organic aerosol through the ozonolysis of $\alpha$-pinene. Synthetic air is simultaneously passed through an ozone-generating lamp and over a small volume of $\alpha$-pinene prior to mixing in a $2.5 \mathrm{~L}$ flow tube where SOA particles form. Ozone is subsequently removed by a charcoal denuder before continuing towards collection onto a microresonator or filter. Flows are controlled by mass flow controllers (MFCs) 
reference filter, respectively. The flow rate for collecting onto the resonator was higher in order to have sufficient flow for impaction. Ozone was produced through the photolysis of oxygen in synthetic air using an ultraviolet lamp (3SC-9, $185 / 254 \mathrm{~nm}, \mathrm{UVP}$ ). The ozone concentration for both collection setups was on the order of $4 \mathrm{ppm}$ as measured by an ozone monitor (Model 49i, Thermo Scientific). Flows were mixed in a $2.5 \mathrm{~L}$ flow tube (where SOA particles form) and passed through a charcoal denuder to remove the remaining volatile organic species and ozone. The method of particle production is shown schematically in Fig. 1. The resulting particle distributions, recorded by scanning mobility particle sizers (SMPSs, Model 3080, TSI Inc.), are compared in Table 1 for the two collection surfaces. While the shape of the particle distribution is similar for both collections (based on the mode diameter), the total mass concentration is 2.5 times higher for collection onto the microresonator. In both cases, the generated particle mass for both collections is significantly higher than ambient levels. However, results of these proof-of-concept experiments comparing particle

Table 1 Comparison of particle distributions produced during collection onto the microresonator and the filter

\begin{tabular}{llll}
\hline Collection surface & $\begin{array}{l}\text { Number mode } \\
\text { diameter } \\
(\mathrm{nm})^{\mathrm{a}}\end{array}$ & $\begin{array}{l}\text { Total mass } \\
\text { concentration } \\
\left(\mathrm{mg} / \mathrm{m}^{3}\right)^{\mathrm{b}, \mathrm{c}}\end{array}$ & $\begin{array}{l}\text { Reaction time } \\
(\mathrm{min})^{\mathrm{d}}\end{array}$ \\
\hline Microresonator & 217 & 179 & 2.1 \\
Filter & 200 & 68 & 6.3 \\
\hline
\end{tabular}

${ }^{\mathrm{a}, \mathrm{b}}$ Number mode diameter and total mass concentration based on SMPS scans

${ }^{\mathrm{c}}$ Mass concentration calculated assuming particle density of $1.2 \mathrm{~g} /$ $\mathrm{cm}^{3}$ [as estimated by (Denjean et al. 2015)] based on scan range of SMPS (14.6-661.2 nm)

${ }^{\mathrm{d}}$ Reaction time based on time before ozone is removed by charcoal denuder composition from filter extraction and MEMS-LESA-MS should not be affected by these high particle concentrations.

The produced SOA was collected either onto a microresonator or a traditional quartz filter using different collection methods. Particle collection onto a microresonator was done using the Microelectromechanical systems (MEMS) Impactor Stage (MIS) using methods described in detail elsewhere (Zielinski et al. 2016) and shown in Fig. 2. To provide context, Fig. 3 includes photographs of the MIS and the microresonator housed within (a detailed image of the resonator itself is included as Fig. 4). In brief, particles are initially size-selected to $300 \mathrm{~nm}$ based on their electric mobility using a differential mobility analyzer (DMA, Model 3081, TSI Inc.) to estimate the expected collected mass based on the difference in reference particle number concentrations upstream and downstream of the MIS as measured using condensation particle counters (CPCs, Models 3775 and 3776, TSI Inc.). Once size-selected, particles are collected onto the microresonator for $1 \mathrm{~h}$ by inertial impaction using a nozzle with $3 \times 0.25 \mathrm{~mm}$ diameter circular jets with a cutoff diameter (the diameter at which $50 \%$ of particles are collected) on the order of $200 \mathrm{~nm}$. Outside of the collection period, two three-way valves are used to control whether the flow contains particles (3WV-1 in Fig. 2) or whether the flow enters the MIS (3WV-2) as part of the overall experimental procedure. For example, while waiting for the ozonegenerating lamp to warm up and the particle concentration to reach steady state in the flow tube, the particle concentration is being measured while bypassing the MIS.

The microresonator is a $1.4 \times 1.4 \mathrm{~mm}$ suspended, piezoelectrically transduced, bulk acoustic resonator described in detail by Prasad et al. (2016) and shown in Fig. 4. It is composed of layers of silicon, silicon oxide, piezoelectric aluminum nitride, and aluminum electrodes with a maximum thickness of $11.7 \mu \mathrm{m}$. The resonator has four triangular electrodes enabling detection of multiple modes although here only the frequency response of the in-plane squareextensional (SE) mode was recorded. The SE mode is

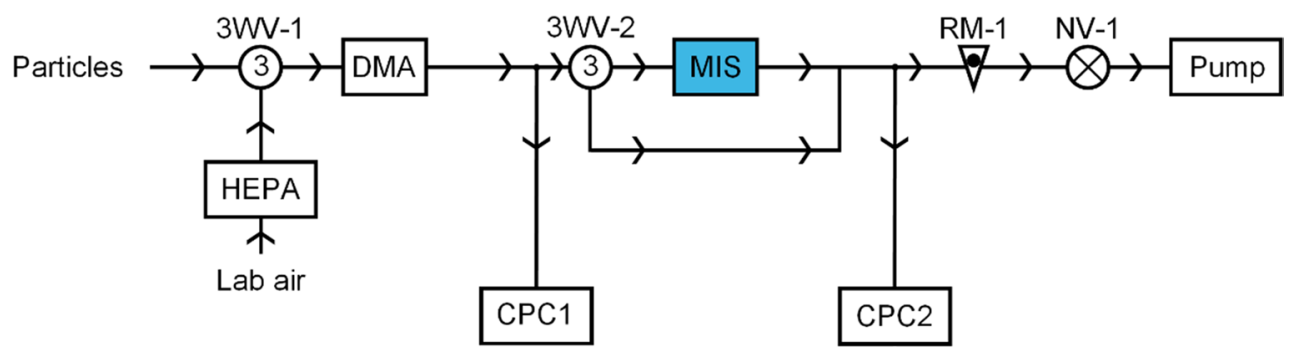

Fig. 2 Flow schematic of the method of collecting particles onto a microresonator housed in the MEMS Impactor Stage (MIS) with black arrows designating flow direction. Two three-way valves (3WV$1,3 \mathrm{WV}-2)$ are used to control the system. During collection, particles are passed through a differential mobility analyzer (DMA, Model 3081, TSI Inc.) to be electrostatically size selected before reaching the MIS. Particles are then collected via inertial impaction in the
MIS. Upstream and downstream particle concentrations are recorded by two condensation particle counters (CPCs, Models 3775 and 3776, TSI Inc.) denoted $\mathrm{CPC} 1$ and $\mathrm{CPC} 2$, respectively. Flow is pulled through the system with a vacuum pump and controlled using a needle valve (NV-1) and rotameter (RM-1). The High Efficiency Particulate Arresting (HEPA) filter is used to flush the system with clean air 

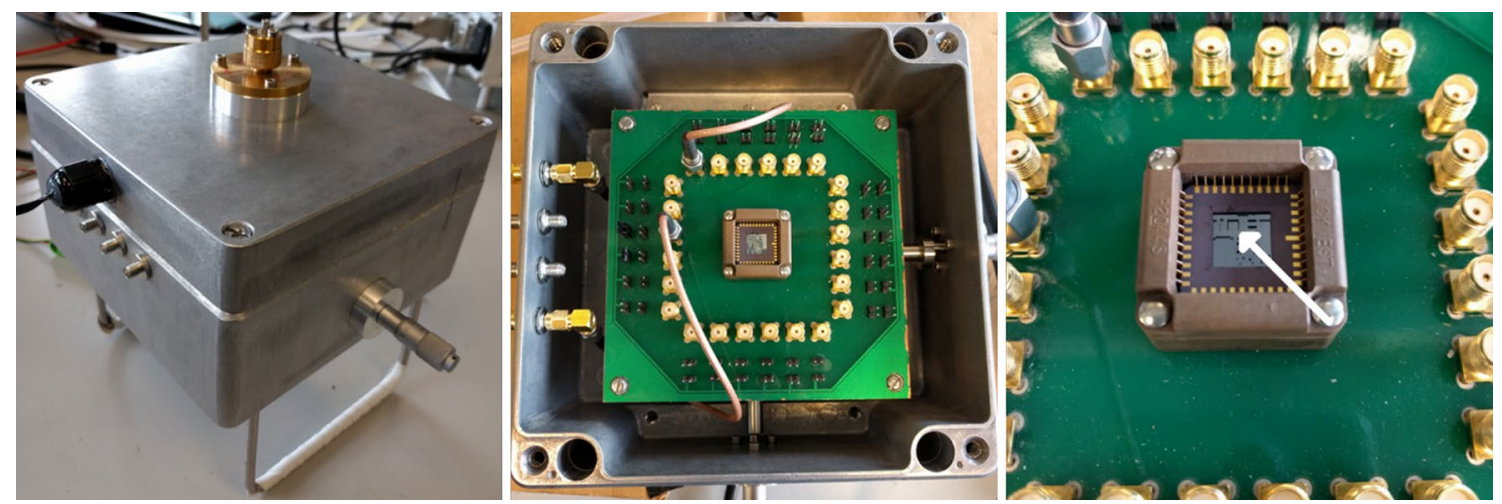

Fig. 3 Representative images of the MIS (left) in its sealed form, (centre) without the lid to highlight chip held within printed circuit board, and (right) a closer image of the chip with the resonator highlighted by an arrow

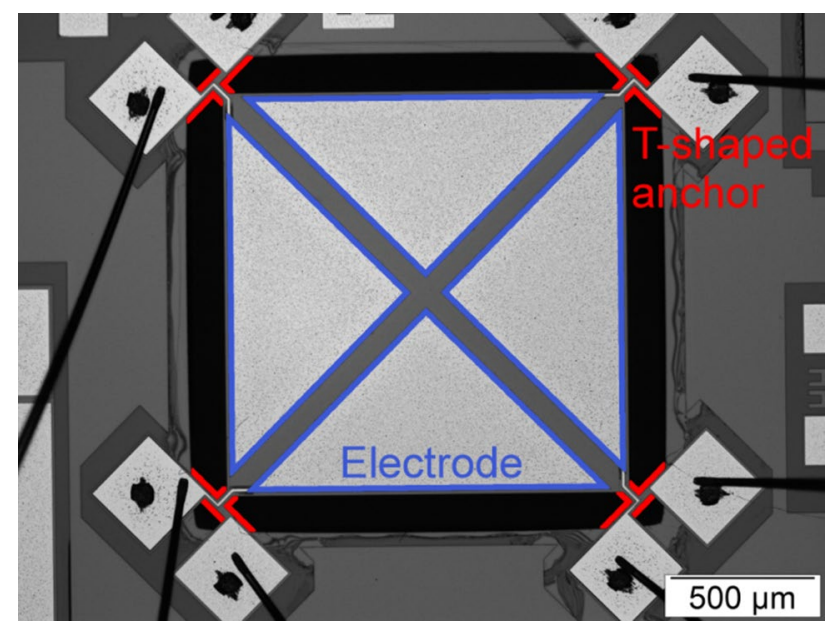

Fig. 4 Image of the resonator front $(1.4 \times 1.4 \mathrm{~mm}$ square $)$ showing four triangular electrodes (highlighted blue, labeled) used to piezoelectrically transduce the resonator. The resonator is suspended by T-shaped anchors at each of the four corners (highlighted red, labeled). Image taken with an optical microscope (BX51, Olympus). Note that aerosol collected on back surface to protect electrodes and electrical connections

characterized by the symmetric extension and contraction of the resonator with a nodal point at its center and has an unloaded (i.e., clean) resonant frequency of $3.16 \mathrm{MHz}$ for the given resonator dimensions. The frequency transmission response (output-to-input ratio) was recorded on 1-min intervals with a network analyzer (N9915A, Agilent) using an output power of $-10 \mathrm{dBm}$ and a span of $20 \mathrm{kHz}$ with 801 measured points (yielding a discrete resolution of $25 \mathrm{~Hz}$ ). The resonant frequency is then determined as the maximum amplitude response for each frequency scan. During the experiment, particles are collected onto the back surface of the resonator (the opposite side of the electrodes) to protect the electronic connections. While the current setup is relatively bulky due to its modular design, future iterations of the impactor can be reduced in size as shown by MaldonadoGarcia et al. (2016), for example. Network analyzers can also be replaced by oscillator circuits for automated measurement of frequency changes (i.e., mass) that are integrated with the resonator (Wasisto et al. 2016; Thomas et al. 2016; Wasisto et al. 2014).

The reference collection was done twice with quartz fiber filters (Pallflex Tissuquartz 2500QAT-UP, $47 \mathrm{~mm}$ diameter) over a span of $2.5 \mathrm{~h}$ each. During collection, the produced SOA was drawn through the filter over the collection period. The expected loading of the filters is roughly $4 \mathrm{mg}$ assuming a density of $1.2 \mathrm{~g} / \mathrm{cm}^{3}$ (Denjean et al. 2015) based on particle size distributions measured with an SMPS and assuming $100 \%$ collection efficiency of the filter.

\subsection{Mass Spectrometry}

The LESA-MS approach begins by aligning a pipette tip containing a small volume (on the order of a few $\mu \mathrm{L}$ ) of the extraction solvent above the sample surface. The solvent is subsequently partially dispensed and brought into contact with the surface while maintaining the liquid junction with the pipette tip. After a prescribed length of time, allowing the sample to dissolve into the solvent, the droplet is aspirated into the pipette tip and sprayed through a nano-electrospray ionization (nanoESI) nozzle into a mass spectrometer. In the case of the microresonator, the pipette must travel past the etched substrate silicon before reaching the device silicon (as shown in Fig. 5) which may otherwise result in wetting of the wrong surface or damaging the chip due to misalignment.

LESA-MS was performed on the resonator surface using methanol (> 99.9\%, Optima ${ }^{\mathrm{TM}}$ LC/MS grade, Fisher Chemical) as the solvent with a total extraction volume of $1.5 \mu \mathrm{L}$. The solvent was partially dispensed $(1.0 \mu \mathrm{L}) 1 \mathrm{~mm}$ above the surface and maintained contact for $1 \mathrm{~s}$. Alignment of the pipette was done manually as the resolution of the 


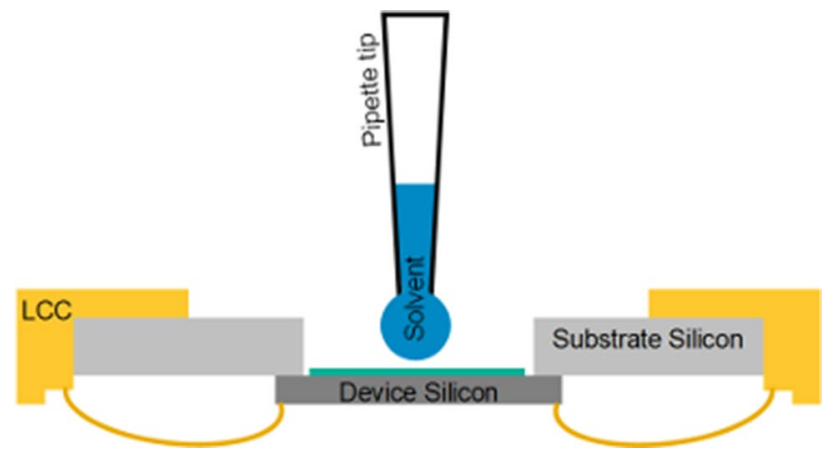

Fig. 5 Schematic of the LESA process for analyzing an aerosol sample (green layer) collected onto a microresonator being accessed through the backside (substrate silicon). The pipette tip must pass through an $8.5 \times 8.5 \mathrm{~mm}$ hole in the leadless chip carrier (LCC) and the etched silicon substrate before reaching the back surface of the device silicon (i.e., the resonator)

instrument $( \pm 2 \mathrm{~mm})$ is not sufficient for automated alignment with the resonator which has a side length of $1.4 \mathrm{~mm}$. The solvent, now containing the dissolved organic aerosol, was infused in a chip-based nanoESI source (TriVersa NanoMate, Advion) and coupled to a high-resolution mass spectrometer (LTQ Velos Orbitrap, Thermo Scientific) which has a resolution of 100,000 at $\mathrm{m} / \mathrm{z}, 400$ and a typical mass accuracy of $\pm 2 \mathrm{ppm}$. The samples were introduced into the mass spectrometer via direct infusion following the procedure (and calibration) as described by Giorio et al. (2015) for negative electrospray ionization. Data were acquired across a $m / z$ range of 100-900 using a lock mass of $m / z 311.16864$ $\left(\mathrm{C}_{17} \mathrm{H}_{27} \mathrm{SO}_{3}{ }^{-}\right)$which is a known contaminant in methanol. Solvent blanks were acquired following the same procedure without dispensing the solvent from the pipette. Each droplet had at least 38 scans acquired (roughly 1 min acquisition time). The procedure was replicated four times for the sample (although only three were fully processed) and three times for the blank. The resonator was imaged before and after the LESA process using an optical microscope (BX51, Olympus) in order to observe the effects of LESA-MS on the resonator surface (e.g., resonator damage and effectiveness of solvent extraction).

Filters were extracted in methanol and analyzed following the procedure already described elsewhere (Kourtchev et al. 2014). Shortly, one eighth of each filter was extracted three times with $5 \mathrm{~mL}$ of methanol (>99.9\%, Optima ${ }^{\mathrm{TM}} \mathrm{LC} /$ MS grade, Fisher Chemical) under ultrasonic agitation in slurry ice for $30 \mathrm{~min}$. The three extracts were combined, filtered through a Teflon ${ }^{\circledR}$ filter $(0.45 \mu \mathrm{m})$ and reduced by volume to approximately $200 \mu \mathrm{L}$ under a gentle stream of nitrogen. Extracts were then analyzed by direct infusion with a nanoESI source (TriVersa Nanomate, Advion) coupled to a high-resolution mass spectrometer (LTQ Velos Orbitrap, Thermo Scientific) across a $m / z$ range of $150-1000$, in two replicates, using the same instrumental settings as for the analysis of the aerosol collected onto the microresonator.

Data treatment of the resulting spectra followed the procedure described by Zielinski et al. (2018). In brief, up to 20 molecular formulae were initially assigned using Xcalibur 2.1 (Thermo Scientific) allowing a maximum mass error of $\pm 4 \mathrm{ppm}$. Formula assignment was based on elemental restrictions of $1 \leq{ }^{12} \mathrm{C} \leq 100,0 \leq{ }^{13} \mathrm{C} \leq 1,1 \leq{ }^{1} \mathrm{H} \leq 200$, $1 \leq{ }^{16} \mathrm{O} \leq 50,0 \leq{ }^{32} \mathrm{~S} \leq 1,0 \leq{ }^{34} \mathrm{~S} \leq 1$. Data were subsequently filtered using an in-house Mathematica 10 (Wolfram Research Inc.) code. The experimental masses were corrected with a mass shift tolerance based on comparing the theoretical and experimental masses of 8 known background contaminants in the spectra. Filtering included only keeping sample peaks with intensities 10 times higher than in the blanks, $\mathrm{O} / \mathrm{C} \leq 2,0.3 \leq \mathrm{H} / \mathrm{C} \leq 2.5, \mathrm{~N} / \mathrm{C} \leq 1.3$, and $\mathrm{S} / \mathrm{C} \leq 0.8$. After processing each individual sample, the final spectrum consisted of peaks found in all sample replicates.

\section{Results and Discussion}

\subsection{Online Mass Sensing}

The online mass measurement of SOA particles during the hour-long collection is given in Fig. 6 as measured by the microresonator (black circles) and the CPCs (pink line, based on the difference in particle number concentrations).

The changing vibrational frequency of the microresonator was converted to a cumulative mass estimate using a theoretical uniform mass sensitivity $(0.034 \mathrm{ng} / \mathrm{Hz})(\mathrm{Zielin}-$ ski et al. 2016). As expected for microresonator mass sensors (Maldonado-Garcia et al. 2016; Paprotny et al. 2013; Lee et al. 2007), the response is linear with time for constant mass addition (based on a constant upstream particle concentration). For comparison purposes, the difference in upstream and downstream CPC particle concentrations was integrated over the collection time to produce the equivalent, cumulative mass assuming a particle diameter of $300 \mathrm{~nm}$ and a density of $1.2 \mathrm{~g} / \mathrm{cm}^{3}$. When plotted in Fig. 6, the resonatorderived mass is scaled by an additional linear factor of 9.1 (determined based on a correlation plot between mass measurements) to be on the same scale as the CPC measurements and is necessary since the difference in CPC particle concentrations is a measure of the total particle loss in the MIS rather than just the collected mass on the resonator. The linear scaling factor accounts for (1) the impactor characteristics of the MIS (i.e., $<100 \%$ collection efficiency of $300 \mathrm{~nm}$ particles causing resonator to underestimate true mass); (2) additional particle loss mechanisms beyond impaction (e.g., impactor wall losses causing resonator to underestimate true mass); (3) collecting larger, multiply charged particles due to electrostatic sizing causing a mismatch between 
Fig. 6 Estimated cumulative mass detected by the microresonator (black circles) and CPCs (pink line) for an approximately constant number concentration of $300 \mathrm{~nm}$ particles (blue line; right y-axis). Low particle concentration at start of collection is before particles have been introduced to the system. Microresonator measurement has been scaled with an additional linear scaling factor of 9.1 as described in detail in the text

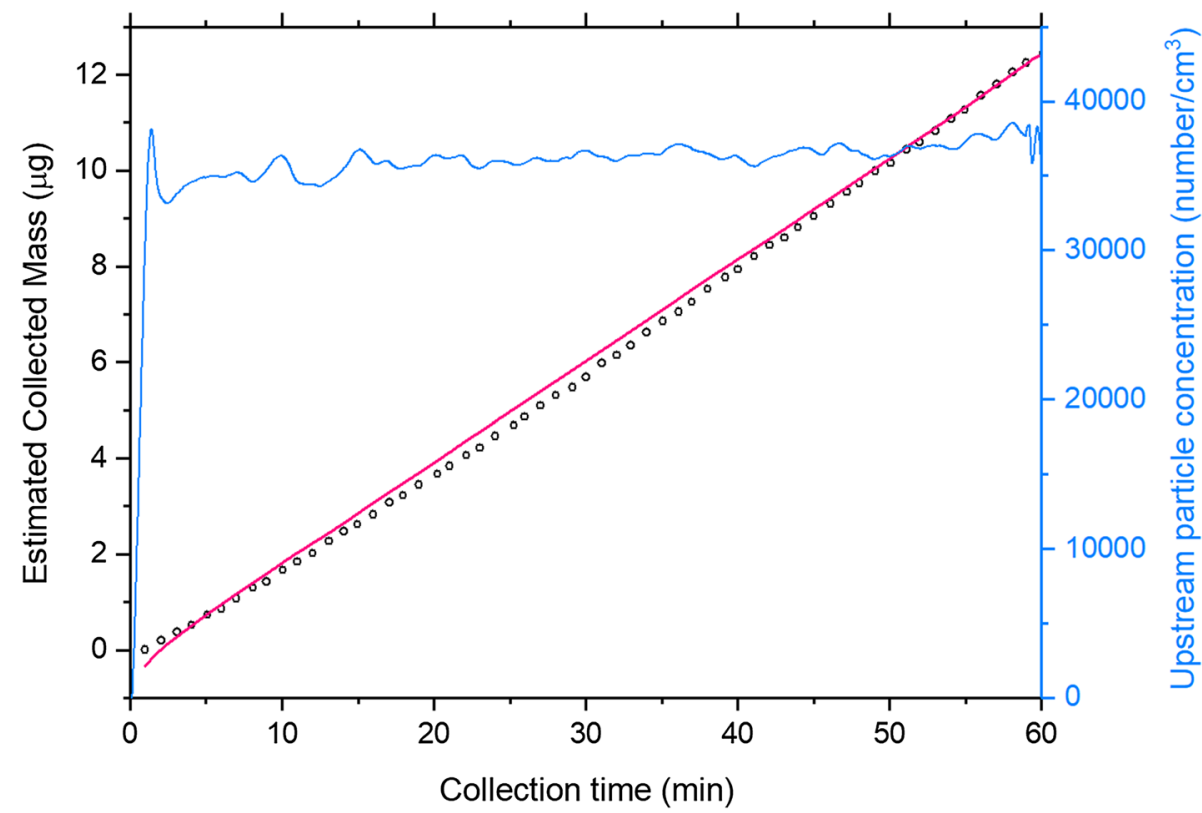

microresonator and CPC estimates; and (4) the spatial sensitivity of the resonator (Zielinski et al. 2015; Campanella et al. 2009) resulting in the frequency response depending on the particle deposition pattern which can both underestimate and overestimate the true mass. These factors are otherwise neglected in a simple frequency-to-mass conversion and serve as a calibration factor for a given particle type and resonator. The actual collected mass on the resonator is expected to be lower than the presented mass in Fig. 6, since it is scaled to match the difference in CPC measurements which account for all particle loss within the MIS (e.g., wall losses). That is, the correction factor of 9.1 used here accounts for all loss mechanisms so the true correction factor (of collected mass only) is less than 9.1. When compared to the mass loading of the filter, the microresonator has roughly 350 times less mass since only monodisperse $300 \mathrm{~nm}$ particles were collected (in addition to the size selection process removing a significant fraction of $300 \mathrm{~nm}$ particles) whereas the filter collected the entire particle distribution. The filter sample remained polydisperse to provide larger particle concentrations given the larger solvent extraction volumes during filter extraction.

After accounting for the linear correction factor, Fig. 6 shows a strong agreement between the two methods of mass measurement (i.e., the microresonator mass follows the trend of the CPC-derived mass) and highlights the capabilities of microresonator mass sensors once the system is calibrated.

A secondary measurement monitored during particle collection is the quality factor (Q-factor). While not explicitly necessary for mass measurement, the Q-factor is a measure of peak "sharpness" and the damping of the resonator. Q-factor is calculated based on the ratio of the resonant frequency to the half-power bandwidth (Logeeswaran et al. 2003). Continuous collection of particles reduces the Q-factor and peak resolution leading to the eventual scenario where the peak cannot be extracted from the data. The mass required to reach this point will vary on the collected aerosol as they will have different damping effects on the resonator. In the case here, the Q-factor went from $~ 1400$ to $\sim 1150$ during collection with no concerns of peak extraction.

\subsection{Integrated Compositional Analysis}

Filter analysis is most commonly employed for aerosol chemical characterization as it allows for specific optimization of the extraction procedure for each aerosol fraction of interest. LESA is a faster, albeit milder, extraction method as extraction occurs only through contact of the sample surface with the solvent. For this reason, extraction efficiency may be limited and needs to be tested.

The resonator surface before and after LESA-MS is shown in Fig. 7 highlighting the cleaning effect of the process and indicating a high extraction efficiency of the deposited SOA. Four "coffee-ring" stains (ring-like particle depositions left after liquid evaporation (Yunker et al. 2011)) remained, however, corresponding to the contact points of the partially deposited solvent droplets from each of the four repeat LESA extractions. The scatter of these contact points highlights a limitation of the current LESA application as the pipette tip would have missed or damaged a smaller resonator but the system can feasibly be optimized to improve its precision. The cleaning of the resonator also limits the number of replicate samples as the collected aerosol is removed with each repetition. The solvent cleaning of the resonator can also be exploited, however, in order to 


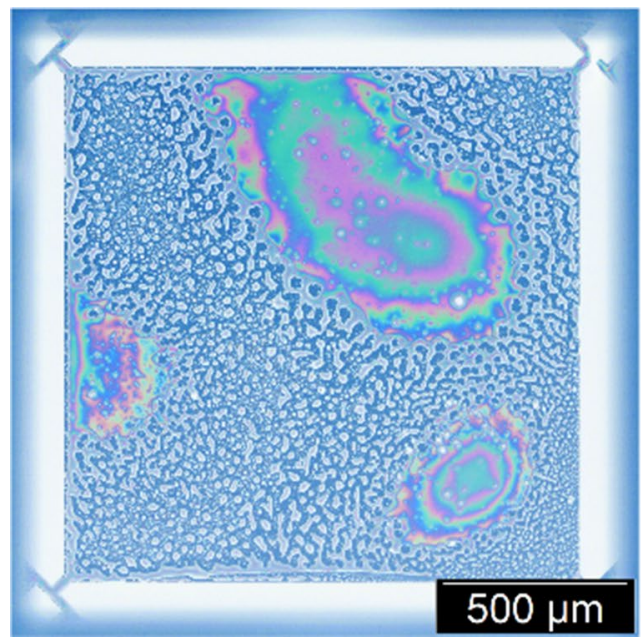

Fig. 7 Collection surface of the resonator (left) after collection and (right) after LESA-MS has been performed four times. Three deposition areas are present after collection with distinct thin-film interference fringes that correspond to the three jets in the impactor noz-

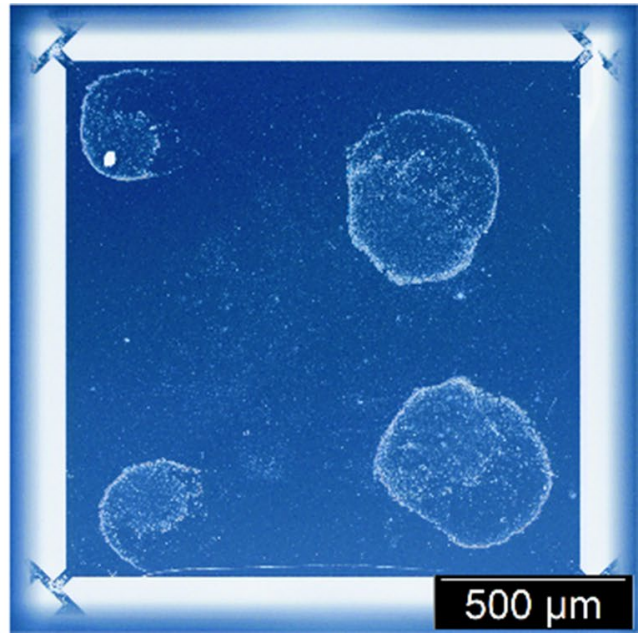

zle. Smaller, speckled collections along the surface correspond to the wider spray from the nozzle. After LESA-MS, the contact point for each sample replicate appears on the resonator as four distinct "coffee-ring" stains in each of the four corners

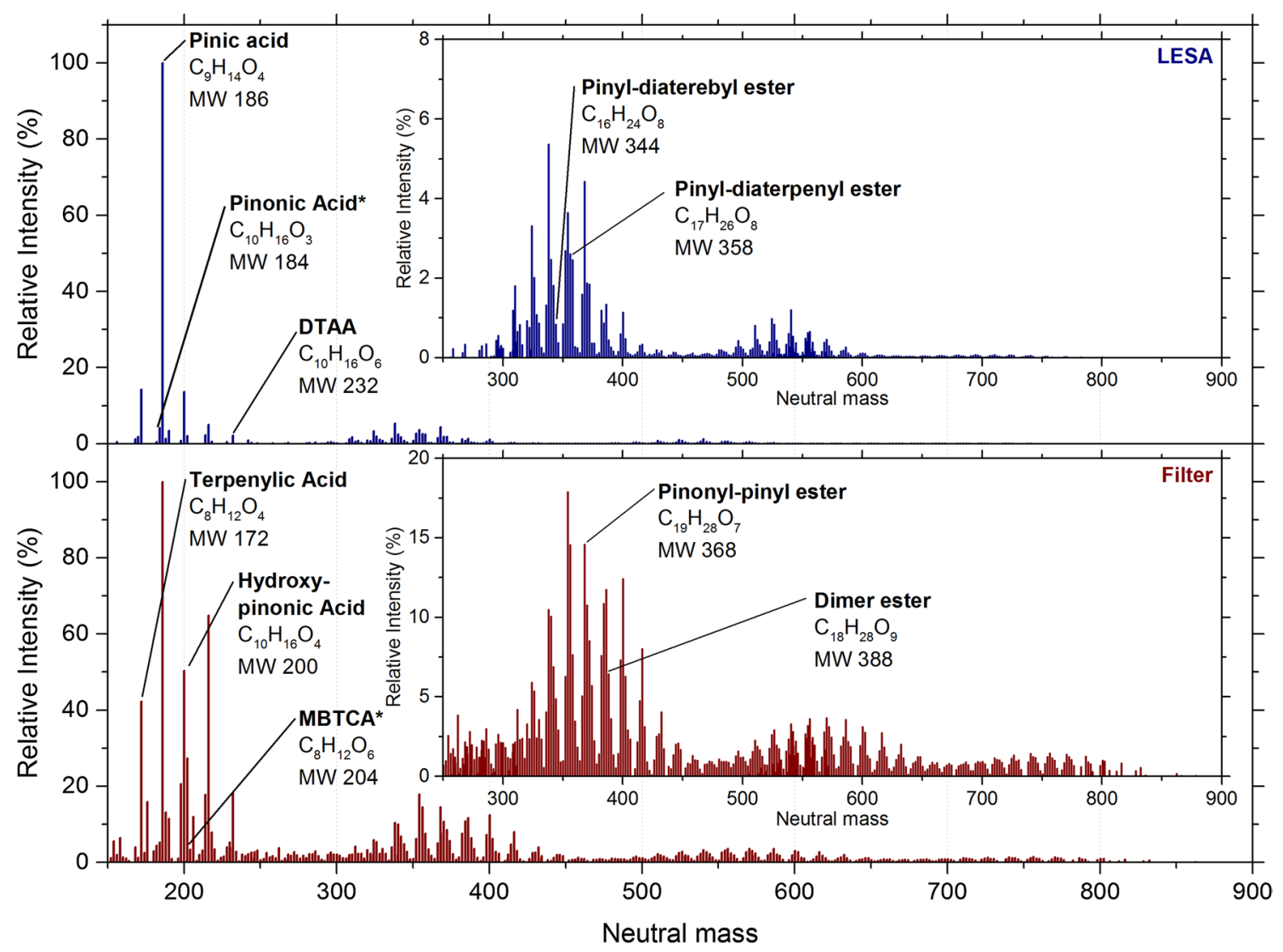

Fig. 8 Mass spectra for LESA (top) and filter (bottom) analyzed through negative electrospray ionization MS. Quoted masses are theoretical neutral masses. Intensities relative to the pinic acid peak (MW 186.08921) that had absolute intensities of $9.3 \times 10^{7}$ and $1.0 \times 10^{7}$ a.u. for LESA and filter, respectively. Labeled monomers and dimers are expected peaks from literature (Kristensen et al. 2014;
Gao et al. 2010; Zhang et al. 2015; Yasmeen et al. 2012) present in both samples unless denoted with an asterisk. Presented $\mathrm{m} / \mathrm{z}$ range (150-900) is for overlapping ranges of LESA (100-900) and filter extraction (150-1000) MS acquisition. DTAA: diaterpenylic acid acetate, MBTCA: Methylbutane-1,2,3-tricarboxylic acid 
reuse the resonator for multiple collections and reduce its effective cost (Zielinski et al. 2017).

The final mass spectra for the microresonator and filter extraction are presented in Fig. 8 based on the peak intensities relative to their most intense peak (pinic acid, $m / z$ 185). The results show a good agreement in the qualitative composition obtained with LESA-MS compared with the traditional filter extraction method followed by analysis with direct infusion nanoESI-MS. Distinct, and expected (Putman et al. 2012), groups of ions (monomers, dimers, and trimers) are visible for both extraction methods in the $\mathrm{m} / \mathrm{z}$ ranges of $<300,300-475$, and 475-650. The filter sample additionally clearly shows a group of tetramers $(\mathrm{m} / \mathrm{z}>650)$, which are hardly visible in the LESA sample. Characteristic products of $\alpha$-pinene ozonolysis are present in both spectra with common monomers and dimers observed in previous studies (Kristensen et al. 2014; Gao et al. 2010; Zhang et al. 2015; Yasmeen et al. 2012) labeled in Fig. 8, including pinic acid, pinonic acid, and hydroxy-pinonic acid. In total, 33\% of the unique assignments across both extraction methods are common. This value is in the same order of magnitude as the common assignments between two filter replicates $(39 \%)$ and includes very low intensity peaks that may not be picked up for each individual repeat MS measurement.
The difference is partially driven by a distinct shift to higher masses (i.e., more oxidised) of dimers and trimers in the filter sample. The cause of the shift is likely the longer reaction time in the flow tube for the filter sample (see Table 1) as it is directly linked with the number of higher mass reaction products. Small differences in ozone and volatile organic compound concentrations will also affect the specific distribution.

Monomer peaks detected with LESA-MS were about an order of magnitude more intense than in the mass spectra of the filter extracts (see Fig. 8 caption), which could be due to a combination of factors: Firstly, although the filter collected more mass ( $\sim 4 \mathrm{mg} v s \sim 12 \mu \mathrm{g}$ ), only an eighth of the filter was extracted and the aerosol on the microresonator was extracted into a much smaller solvent volume (i.e., $200 \mu \mathrm{L}$ for filter extraction vs. $1.5 \mu \mathrm{L}$ for LESA). Therefore, if we assume that all aerosol was removed after four LESA extractions (as shown qualitatively in Fig. 7), the concentrations of aerosol components in the filter extracts $(\sim 2.5 \mathrm{~g} / \mathrm{L})$ are close to those of the LESA samples $(\sim 2 \mathrm{~g} / \mathrm{L})$. However, based on the reducing signal intensities by the third LESA sample (Fig. 9), it is more likely that the concentration of the LESA samples was initially higher than the filter but finished lower due to the cleaning effect of the procedure.

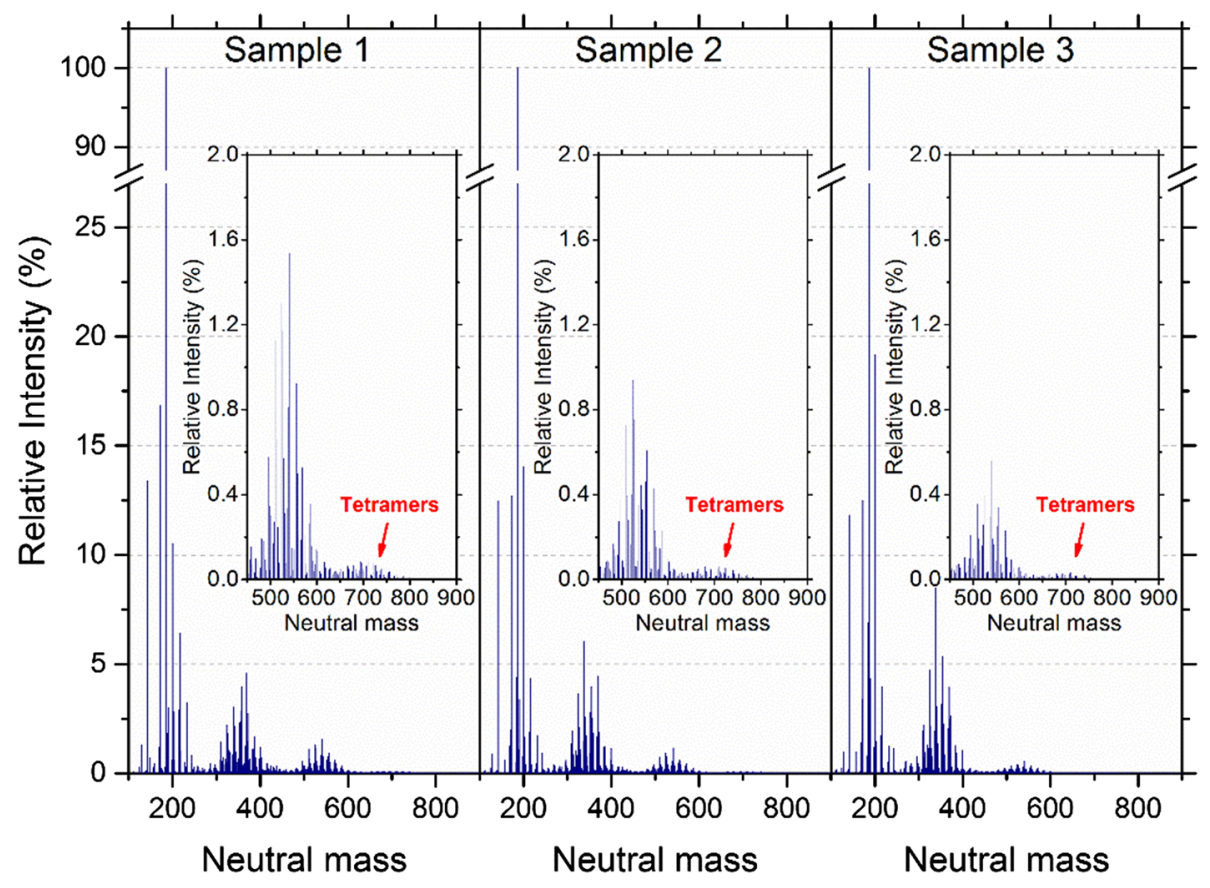

Fig. 9 Mass spectra for the three LESA-MS samples showing very similar spectra for the first two extractions but reduced intensities by the third sample for trimers and tetramers. Intensities are presented relative to the highest intensity peak of each sample (pinic acid, MW 186.08921 ) with the absolute intensities of pinic acid being $1.0 \times 10^{8}$, $1.2 \times 10^{8}$, and $0.5 \times 10^{8}$ for Samples 1, 2, and 3, respectively. As shown in the insets, the intensity reduction of the third sample is particularly evident in the trimers and tetramers (MW $>475)$ with tetramers nearly disappearing by the third sample (as highlighted). This results in the final spectrum, which only considers ions common across all samples, having only a few, low intensity tetramers. Quoted masses values are theoretical neutral masses and are presented across the full scan span for LESA-MS (100-900) 
Secondly, it may be argued that solvent evaporation during filter sample preparation promotes losses of lower molecular weight/more volatile species and the formation of oligomers as it happens in evaporating water droplets (Galloway et al. 2014). Overall this comparison shows the much higher sensitivity of LESA analyses compared to conventional filter sampling and extraction procedures.

In contrast, the final intensity of the LESA spectrum is lowered by the common ion selection processing method since the final intensities are averaged across three replicates. Since the liquid extraction method cleaned the resonator, as shown in Fig. 7, each subsequent sample has less aerosol to extract resulting in lower intensities and lower numbers of compounds $(2738,2362,1579)$. The individual spectra for each LESA sample are shown in Fig. 9. The cleaning effect of LESA-MS also largely removed the presence of tetramers from the final, combined spectrum although they are present in the first two samples. It should be noted that the same common ion selection method was used with the filter samples but the replicates had consistent spectra as the extraction solution was the same for both filter replicates.

Quantitatively, the final LESA-MS spectrum (including only ions common across three replicates) has $22 \%$ of the unique assignments from each individual replicate. In contrast, $43 \%$ of the ions are common across the first two replicates. This is similar to the value across the two filter replicates that had $39 \%$ common ions.

Future application of the system to ambient applications will require careful consideration of the influence of environmental factors on the microresonator. For example, the resonator response to temperature changes is well known (Jha et al. 2007) and will need to be corrected for. Potential methods include post-processing using a known linear correction factor, using a reference resonator, or controlling the temperature with a micro-hotplate (Udrea et al. 2001). Another concern is humidity which can greatly influence the detected mass of the aerosol. The most reliable method to remove the influence of humidity would be to dry particles before collection (like many commercial monitoring stations). The MIS also offers the potential of determining the hygroscopicity of the collected aerosol afterwards through controlled humidity ramping (Zielinski et al. 2018). The influence of environmental factors will also need to be addressed for the associated electronics, such as oscillator circuits, implemented for automated recording.

\section{Conclusions}

A new approach of combining the high time resolution of a microresonator with the integrated compositional analysis of LESA-MS was introduced. The performance of this methodology was compared to the conventional filter sampling approach in the analysis of secondary organic aerosol produced from the ozonolysis of $\alpha$-pinene.

In particular, the current arrangement offers significantly improved time resolution over offline filter methods (i.e., 1 min vs. hours or longer) due to the high mass sensitivity of the microresonator. Implementing an oscillator circuit would provide automated recording at costs that, for mass production, have the potential to be comparable to traditional filter-based methods with the additional advantage that the microresonator can be reused. Concerning chemical analysis, the LESA-MS approach applied to the microresonator is significantly less time consuming than traditional filter extraction methods and provides a sensitivity that is at least as good. In fact, the aerosol particles are deposited on a small surface area in the microresonator so that more aerosol mass is available for a single droplet extraction and analysis. Additional environmental factors will need to be controlled, however, before reliable ambient measurements can be performed.

Despite minor differences between the two presented MS methods, LESA-MS in tandem with microresonators is an option for future compositional analysis with online mass measurements. The method can be further exploited by arranging microresonators in a small-scale, portable cascade impactor arrangement (Maldonado-Garcia et al. 2016) to provide size-segregated online mass and integrated bulk compositional information of the collected sample. Future microresonator surface compositional analysis with mass spectrometry could be extended to smaller resonators in one of two ways. Firstly, using DESI instead of LESA may allow accessibility for smaller resonators because of its smaller desorption area (Takáts et al. 2004). This method is characterized by a continuous electrospray being directed at a sample with the desorbed ions being directly sampled by a mass spectrometer. One major requirement is an insulating surface (or sample) such as polytetrafluoroethylene (PTFE) coating (Takáts et al. 2004) which can be deposited as thin films on microresonators (Bodas and Gangal 2005). Conversely, in contrast to LESA, DESI does not allow controlled extraction time (Giorio et al. 2015) posing challenges when analyzing less readily soluble aerosol such as ambient aerosol. A second, cruder approach is to rinse the resonator with a solvent and produce a mass spectrum using the rinsings. This method would allow for multiple, uniform replicates, but is more laborious and susceptible to contamination. Regardless of the chosen MS method, this work highlights the potential for extending the application of microresonator particulate mass sensors beyond mass measurements to include chemical analysis. 
Acknowledgements This work was funded by the European Research Council (ERC starting grant 279405), the UK Natural Environment Research Council (grant NE/K008218/1). ATZ thanks the Natural Sciences and Engineering Research Council of Canada, the Sir Winston Churchill Society of Edmonton, and the Cambridge Trust for funding of the $\mathrm{PhD}$ degree.

\section{Compliance with ethical standards}

Conflict of interest On behalf of all authors, the corresponding author states that there is no conflict of interest.

Open Access This article is distributed under the terms of the Creative Commons Attribution 4.0 International License (http://creativeco mmons.org/licenses/by/4.0/), which permits unrestricted use, distribution, and reproduction in any medium, provided you give appropriate credit to the original author(s) and the source, provide a link to the Creative Commons license, and indicate if changes were made.

\section{References}

Abdolvand R, Lavasani H, Ho G, Ayazi F (2008) Thin-film piezoelectric-on-silicon resonators for high-frequency reference oscillator applications. IEEE Trans Ultrason Ferroelectr Freq Control 55(12):2596-2606. https://doi.org/10.1109/TUFFC.2008.976

Allen G, Sioutas C, Koutrakis P, Reiss R, Lurmann FW, Roberts PT (1997) Evaluation of the TEOM ${ }^{\circledR}$ method for measurement of ambient particulate mass in Urban areas. J Air Waste Manage Assoc 47(6):682-689. https://doi.org/10.1080/10473 289.1997.10463923

Amato F, Alastuey A, Karanasiou A, Lucarelli F, Nava S, Calzolai G, Severi M, Becagli S, Gianelle VL, Colombi C et al (2016) AIRUSE-LIFE + : a harmonized PM speciation and source apportionment in five southern European cities. Atmos Chem Phys 16(5):3289-3309. https://doi.org/10.5194/acp-16-3289-2016

Bateman AP, Walser ML, Desyaterik Y, Laskin J, Laskin A, Nizkorodov SA (2008) The effect of solvent on the analysis of secondary organic aerosol using electrospray ionization mass spectrometry. Environ Sci Technol 42(19):7341-7346. https://doi.org/10.1021/ es801226w

Bodas DS, Gangal SA (2005) PTFE as a masking material for MEMS fabrication. J Micromechanics Microeng 15:802-806. https://doi. org/10.1088/0960-1317/15/4/018

Broday DM, the Citi-Sense Project Collaborators (2017) Wireless distributed environmental sensor networks for air pollution measurement-the promise and the current reality. Sensors 17(10):2263. https://doi.org/10.3390/s17102263

Campanella H, Martincic E, Nouet P, Uranga A, Esteve J (2009) Analytical and finite-element modeling of localized-mass sensitivity of thin-film bulk. IEEE Sens J 9(8):892-901. https://doi. org/10.1109/JSEN.2009.2024858

Chen H, Li M, Zhang Y-P, Yang X, Lian J-J, Chen J-M (2008) Rapid analysis of svoc in aerosols by desorption electrospray ionization mass spectrometry. J Am Soc Mass Spectrom 19(3):450-454. https://doi.org/10.1016/j.jasms.2007.11.020

Chow JC, Watson JG (2007) Review of measurement methods and compositions for ultrafine particles. Aerosol Air Qual Res 7(2):121-173. https://doi.org/10.4209/aaqr.2007.05.0029

Denjean C, Formenti P, Picquet-Varrault B, Pangui E, Zapf P, Katrib Y, Giorio C, Tapparo A, Monod A, Temime-Roussel B et al (2015) Relating hygroscopicity and optical properties to chemical composition and structure of secondary organic aerosol particles generated from the ozonolysis of $\alpha$-pinene. Atmos Chem Phys 15(6):3339-3358. https://doi.org/10.5194/acp-15-3339-2015

European Parliament; Council of the European Union (2008) Directive 2008/50/EC of the European parliament and of the council of 21 May 2008 on ambient air quality and cleaner air for Europe. Off J Eur Union 51(152):1-44

Fuller SJ, Zhao Y, Cliff SS, Wexler AS, Kalberer M (2012) Direct surface analysis of time-resolved aerosol impactor samples with ultrahigh-resolution mass spectrometry. Anal Chem 84(22):98589864. https://doi.org/10.1021/ac3020615

Galloway MM, Powelson MH, Sedehi N, Wood SE, Millage KD, Kononenko JA, Rynaski AD, De Haan DO (2014) Secondary organic aerosol formation during evaporation of droplets containing atmospheric aldehydes, amines, and ammonium sulfate. Environ Sci Technol 48(24):14417-14425. https://doi. org/10.1021/es5044479

Gao Y, Hall WA IV, Johnston MV (2010) Molecular composition of monoterpene secondary organic aerosol at low mass loading. Environ Sci Technol 44(20):7897-7902. https://doi. org/10.1021/es101861k

Giorio C, Moyroud E, Glover BJ, Skelton PC, Kalberer M (2015) Direct surface analysis coupled to high-resolution mass spectrometry reveals heterogeneous composition of the cuticle of Hibiscus trionum petals. Anal Chem 87(19):9900-9907. https ://doi.org/10.1021/acs.analchem.5b02498

Grimm H, Eatough DJ (2009) aerosol measurement: the use of optical light scattering for the determination of particulate size distribution, and particulate mass, including the semi-volatile fraction. J Air Waste Manage Assoc 9(1):101-107. https://doi. org/10.3155/1047-3289.59.1.101

Harrison RM, Yin J (2000) Particulate matter in the atmosphere: which particle properties are important for its effects on health? Sci Total Environ 249(1-3):85-101. https://doi.org/10.1016/ S0048-9697(99)00513-6

Harrison RM, Giorio C, Beddows DCS (2010) Dall'Osto, M. Size distribution of airborne particles controls outcome of epidemiological studies. Sci Total Environ 409(2):289-293. https://doi. org/10.1016/j.scitotenv.2010.09.043

Heim M, Mullins BJ, Umhauer H, Kasper G (2008) Performance evaluation of three optical particle counters with an efficient "multimodal" calibration method. J Aerosol Sci 39(12):1019_ 1031. https://doi.org/10.1016/j.jaerosci.2008.07.006

Holstius DM, Pillarisetti A, Smith KR, Seto E (2014) Field calibrations of a low-cost aerosol sensor at a regulatory monitoring site in California. Atmos Meas Tech 7(4):1121-1131. https:// doi.org/10.5194/amt-7-1121-2014

Jha CM, Bahl G, Melamud R, Chandorkar SA, Hopcroft MA, Kim B, Agarwal M, Salvia J, Mehta H, Kenny TW (2007) High resolution microresonator-based digital temperature sensor. Appl Phys Lett 91(7):74101. https://doi.org/10.1063/1.2768629

Kourtchev I, Fuller S, Aalto J, Ruuskanen TM, McLeod MW, Maenhaut W, Jones R, Kulmala M, Kalberer M (2013) Molecular composition of boreal forest aerosol from Hyytiälä, Finland, using ultrahigh resolution mass spectrometry. Environ Sci Technol 47(9):4069-4079. https://doi.org/10.1021/es3051636

Kourtchev I, Fuller SJ, Giorio C, Healy RM, Wilson E, O'Connor I, Wenger JC, McLeod M, Aalto J, Ruuskanen TM et al (2014) Molecular composition of biogenic secondary organic aerosols using ultrahigh-resolution mass spectrometry: comparing laboratory and field studies. Atmos Chem Phys 14(4):2155-2167. https://doi.org/10.5194/acp-14-2155-2014

Kristensen K, Cui T, Zhang H, Gold A, Glasius M, Surratt JD (2014) Dimers in $\alpha$-pinene secondary organic aerosol: effect of hydroxyl radical, ozone, relative humidity and aerosol acidity. Atmos Chem Phys 14(8):4201-4218. https://doi.org/10.5194/ acp-14-4201-2014 
Laskin J, Laskin A, Roach PJ, Slysz GW, Anderson GA, Nizkorodov SA, Bones DL, Nguyen LQ (2010) High-resolution desorption electrospray ionization mass spectrometry for chemical characterization of organic aerosols. Anal Chem 82(5):2048-2058. https ://doi.org/10.1021/ac902801f

Lee JE-Y, Bahreyni B, Zhu Y, Seshia AA (2007) Ultrasensitive mass balance based on a bulk acoustic mode single-crystal silicon resonator. Appl Phys Lett 91(23):89-92. https://doi. org/10.1063/1.2822405

Li M, Chen H, Yang X, Chen J, Li C (2009a) Direct quantification of organic acids in aerosols by desorption electrospray ionization mass spectrometry. Atmos Environ 43(17):2717-2720. https://doi. org/10.1016/j.atmosenv.2009.02.057

Li M, Chen H, Wang BF, Yang X, Lian JJ, Chen JM (2009b) Direct quantification of PAHs in biomass burning aerosols by desorption electrospray ionization mass spectrometry. Int J Mass Spectrom 281(1-2):31-36. https://doi.org/10.1016/j.ijms.2008.11.013

Logeeswaran VJ, Tay FEH, Chan ML, Chau FS, Liang YC (2003) First harmonic (2f) characterisation of resonant frequency and Q-factor of micromechanical transducers. Analog Integr Circuits Signal Process 37(1):17-33. https://doi.org/10.1023/A:1024816600152

Maldonado-Garcia M, Kumar V, Wilson JC, Pourkamali S (2016) Chipscale implementation and cascade assembly of particulate matter collectors with embedded resonant mass balances. IEEE Sens J 17(6):1617-1625. https://doi.org/10.1109/JSEN.2016.2638964

Mazzoleni LR, Saranjampour P, Dalbec MM, Samburova V, Hallar AG, Zielinska B, Lowenthal DH, Kohl S (2012) Identification of water-soluble organic carbon in non-urban aerosols using ultrahigh-resolution FT-ICR mass spectrometry: organic anions. Environ Chem 9(3):285. https://doi.org/10.1071/EN11167

Mehdizadeh E, Kumar VS, Wilson J, Pourkamali S (2017) Inertial impaction on MEMS balance chips for real-time air quality monitoring. IEEE Sens J 17(8):2329-2337. https://doi.org/10.1109/ JSEN.2017.2675958

Miljevic B, Hedayat F, Stevanovic S, Fairfull-Smith KE, Bottle SE, Ristovski ZD (2014) To sonicate or not to sonicate PM filters: reactive oxygen species generation upon ultrasonic irradiation. Aerosol Sci Technol 48(12):1276-1284. https://doi. org/10.1080/02786826.2014.981330

Nguyen TB, Laskin J, Laskin A, Nizkorodov SA (2011) Nitrogen-containing organic compounds and oligomers in secondary organic aerosol formed by photooxidation of isoprene. Environ Sci Technol 45(16):6908-6918. https://doi.org/10.1021/es201611n

O'brien RE, Nguyen TB, Laskin A, Laskin J, Hayes PL, Liu S, Jimenez JL, Russell LM, Nizkorodov SA, Goldstein AH (2013) Probing molecular associations of field-collected and laboratorygenerated soa with nano-desi high-resolution mass spectrometry. J Geophys Res Atmos 118(2):1042-1051. https://doi.org/10.1002/jgrd.50119

Ohno T, Sleighter RL, Hatcher PG (2016) Comparative study of organic matter chemical characterization using negative and positive mode electrospray ionization ultrahigh-resolution mass spectrometry. Anal Bioanal Chem 408(10):2497-2504. https:// doi.org/10.1007/s00216-016-9346-x

Paprotny I, Doering F, Solomon PA, White RM, Gundel LA (2013) Microfabricated air-microfluidic sensor for personal monitoring of airborne particulate matter: design, fabrication, and experimental results. Sensors Actuators A Phys 201:506-516. https://doi. org/10.1016/j.sna.2012.12.026

Piazza G, Stephanou PJ, Pisano AP (2007) Single-chip multiple-frequency ALN MEMS filters based on contour-mode piezoelectric resonators. J. Microelectromechanical Syst 16(2):319-328. https ://doi.org/10.1109/JMEMS.2006.889503

Prasad A, Charmet J, Seshia AA (2016) Simultaneous interrogation of high-Q modes in a piezoelectric-on-silicon micromechanical resonator. Sensors Actuators A Phys 238:207-214. https://doi. org/10.1016/j.sna.2015.12.013
Putman AL, Offenberg JH, Fisseha R, Kundu S, Rahn TA, Mazzoleni LR (2012) Ultrahigh-resolution FT-ICR mass spectrometry characterization of $\alpha$-pinene ozonolysis SOA. Atmos Environ 46:164-172. https://doi.org/10.1016/j.atmosenv.2011.10.003

Raaschou-Nielsen O, Andersen ZJ, Beelen R, Samoli E, Stafoggia M, Weinmayr G, Hoffmann B, Fischer P, Nieuwenhuijsen MJ, Brunekreef B et al (2013) Air pollution and lung cancer incidence in 17 European cohorts: prospective analyses from the European study of cohorts for air pollution effects (ESCAPE). Lancet Oncol 14(9):813-822. https://doi.org/10.1016/S1470-2045(13)70279-1

Romonosky DE, Laskin A, Laskin J, Nizkorodov SA (2014) Highresolution mass spectrometry and molecular characterization of aqueous photochemistry products of common types of secondary organic aerosols. J Phys Chem A 119:2594-2606. https://doi. org/10.1021/jp509476r

Steinle S, Reis S, Sabel CE (2013) Quantifying human exposure to air pollution-Moving from static monitoring to spatio-temporally resolved personal exposure assessment. Sci Total Environ 443:184-193. https://doi.org/10.1016/j.scitotenv.2012.10.098

Steinle S, Reis S, Sabel CE, Semple S, Twigg MM, Braban CF, Leeson SR, Heal MR, Harrison D, Lin C et al (2015) Personal exposure monitoring of PM2.5 in indoor and outdoor microenvironments. Sci Total Environ 508:383-394. https://doi.org/10.1016/j.scito tenv.2014.12.003

Takáts Z, Wiseman JM, Gologan B, Cooks RG (2004) Mass spectrometry sampling under ambient conditions with desorption electrospray ionization. Science (80-). 306(5695):471-473. https://doi. org/10.1126/science.1104404

Thomas S, Cole M, Villa-López FH, Gardner JW (2016) High frequency surface acoustic wave resonator-based sensor for particulate matter detection. Sensors Actuators A Phys. 244:138-145. https://doi.org/10.1016/j.sna.2016.04.003

Udrea F, Gardner JW, Setiadi D, Covington JA, Dogaru T, Lu CC, Milne WI (2001) Design and simulations of SOI CMOS microhotplate gas sensors. Sensors Actuators B Chem 78(1-3):180190. https://doi.org/10.1016/s0925-4005(01)00810-3

Wang Y, Li J, Jing H, Zhang Q, Jiang J, Biswas P (2015) Laboratory evaluation and calibration of three low-cost particle sensors for particulate matter measurement. Aerosol Sci Technol 49(11):1063-1077. https://doi.org/10.1080/02786826.2015.11007 10

Wang XK, Rossignol S, Ma Y, Yao L, Wang MY, Chen JM, George C, Wang L (2016) Molecular characterization of atmospheric particulate organosulfates in three megacities at the middle and lower reaches of the Yangtze River. Atmos Chem Phys 16(4):22852298. https://doi.org/10.5194/acp-16-2285-2016

Wasisto HS, Merzsch S, Waag A, Uhde E, Salthammer T, Peiner E (2013a) Evaluation of photoresist-based nanoparticle removal method for recycling silicon cantilever mass sensors. Sensors Actuators A Phys 202:90-99. https://doi.org/10.1016/j. sna.2012.12.016

Wasisto HS, Merzsch S, Stranz A, Waag A, Uhde E, Salthammer T, Peiner E (2013b) Silicon resonant nanopillar sensors for airborne titanium dioxide engineered nanoparticle mass detection. Sensors Actuators B Chem 189:146-156. https://doi.org/10.1016/j. snb.2013.02.053

Wasisto HS, Zhang Q, Merzsch S, Waag A, Peiner E (2014) A phaselocked loop frequency tracking system for portable microelectromechanical piezoresistive cantilever mass sensors. Microsyst Technol 20(4-5):559-569. https://doi.org/10.1007/s0054 2-013-1991-9

Wasisto HS, Merzsch S, Uhde E, Waag A, Peiner E (2015) Partially integrated cantilever-based airborne nanoparticle detector for continuous carbon aerosol mass concentration monitoring. J Sensors Sens Syst 4(1):111-123. https://doi.org/10.5194/jsss-4-111-2015 
Wasisto HS, Uhde E, Peiner E (2016) Enhanced performance of pocket-sized nanoparticle exposure monitor for healthy indoor environment. Build Environ 95:13-20. https://doi.org/10.1016/j. buildenv.2015.09.013

Xing YF, Xu YH, Shi MH, Lian YX (2016) The impact of PM2.5 on the human respiratory system. J. Thorac. Dis 8(1):69-74. https:// doi.org/10.3978/j.issn.2072-1439.2016.01.19

Yasmeen F, Vermeylen R, Maurin N, Perraudin E, Doussin J-F, Claeys $M$ (2012) Characterisation of tracers for aging of $\alpha$-pinene secondary organic aerosol using liquid chromatography/negative ion electrospray ionisation mass spectrometry. Environ Chem 9(3):236-246. https://doi.org/10.1071/EN11148

Yunker PJ, Still T, Lohr MA, Yodh AG (2011) Suppression of the coffee-ring effect by shape-dependent capillary interactions. Nature 476(7360):308-311. https://doi.org/10.1038/nature10344

Zhang X, McVay RC, Huang DD, Dalleska NF, Aumont B, Flagan RC, Seinfeld JH (2015) Formation and evolution of molecular products in $\alpha$-pinene secondary organic aerosol. Proc Natl Acad Sci 112(46):14168-14173. https://doi.org/10.1073/pnas.1517742112

Zielinski AT, Kalberer M, Jones RL, Prasad A, Seshia AA (2016) Particulate mass sensing with piezoelectric bulk acoustic mode resonators. In: Internationl Frequency Control Symposium (IFCS), IEEE, LA, pp 1-6

Zielinski AT, Prasad A, Seshia AA, Kalberer M, Jones RL (2015) Effects of spatial sensitivity on mass sensing with bulk acoustic mode resonators. Sensors Actuators A Phys 236:369-379. https ://doi.org/10.1016/j.sna.2015.11.003

Zielinski AT, Weckman NE, Jones RL, Kalberer M, Seshia AA (2017) Extending the lifetime of resonant atmospheric particulate mass sensors with solvent rinses. IEEE Sensors Lett 1(5):1-4. https:// doi.org/10.1109/1sens.2017.2734569

Zielinski AT, Kourtchev I, Bortolini C, Fuller SJ, Giorio C, Popoola OAM, Bogialli S, Tapparo A, Jones RL, Kalberer M (2018a) A new processing scheme for ultra-high resolution direct infusion mass spectrometry data. Atmos Environ 178:129-139. https://doi. org/10.1016/j.atmosenv.2018.01.034

Zielinski AT, Gallimore PJ, Griffiths PT, Jones RL, Seshia AA, Kalberer M (2018b) Measuring aerosol phase changes and hygroscopicity with a microresonator mass sensor. Anal, Chem in press

Zou X, Thiruvenkatanathan P, Seshia AA (2014) A seismic-grade resonant MEMS accelerometer. J. Microelectromechanical Syst 23(4):768-770. https://doi.org/10.1109/jmems.2014.2319196 